\title{
Temperature-dependent phonon lifetimes and thermal conductivity of silicon by inelastic neutron scattering and $a b$ initio calculations
}

\author{
D. S. Kim $\odot,{ }^{1, *}$ O. Hellman, ${ }^{1}$ N. Shulumba, ${ }^{2}$ C. N. Saunders $\odot,{ }^{1}$ J. Y. Y. Lin, ${ }^{3}$ H. L. Smith,${ }^{4}$ J. E. Herriman $\odot,{ }^{1}$ J. L. Niedziela, ${ }^{5}$ \\ D. L. Abernathy ${ }^{6},{ }^{6}$ C. W. $\mathrm{Li}^{7}{ }^{7}$ and B. Fultz ${ }^{1, \dagger}$ \\ ${ }^{1}$ Department of Applied Physics and Materials Science, California Institute of Technology, Pasadena, California 91125, USA \\ ${ }^{2}$ Department of Mechanical and Civil Engineering, California Institute of Technology, Pasadena, California 91125, USA \\ ${ }^{3}$ Neutron Data Analysis and Visualization Division, Oak Ridge National Laboratory, Oak Ridge, Tennessee 37831, USA \\ ${ }^{4}$ Physics and Astronomy Department, Swarthmore University, Swarthmore, Pennsylvania 19081, USA \\ ${ }^{5}$ Division of Materials Science and Technology, Oak Ridge National Laboratory, Oak Ridge, Tennessee 37831, USA \\ ${ }^{6}$ Division of Neutron Scattering, Oak Ridge National Laboratory, Oak Ridge, Tennessee 37831, USA \\ ${ }^{7}$ Department of Mechanical Engineering, University of California, Riverside, California 92521, USA
}

(Received 16 June 2020; revised 8 October 2020; accepted 27 October 2020; published 16 November 2020)

\begin{abstract}
Inelastic neutron scattering on a single crystal of silicon was performed at temperatures from 100 to $1500 \mathrm{~K}$. These experimental data were reduced to obtain phonon spectral intensity at all wave vectors $\vec{Q}$ and frequencies $\omega$ in the first Brillouin zone. Thermal broadenings of the phonon peaks were obtained by fitting and by calculating with an iterative $a b$ initio method that uses thermal atom displacements on an ensemble of superlattices. Agreement between the calculated and experimental broadenings was good, with possible discrepancies at the highest temperatures. Distributions of phonon widths versus phonon energy had similar shapes for computation and experiment. These distributions grew with temperature but maintained similar shapes. Parameters from the $a b$ initio calculations were used to obtain the thermal conductivity from the Boltzmann transport equation, which was in good agreement with experimental data. Despite the high group velocities of longitudinal acoustic phonons, their shorter lifetimes reduced their contribution to the thermal conductivity, which was dominated by transverse acoustic modes.
\end{abstract}

DOI: 10.1103/PhysRevB.102.174311

\section{INTRODUCTION}

Phonons underly the thermophysical properties of materials. The spectrum of energies of phonons, i.e., the phonon density of states (DOS), can provide the phonon partition function, free energy, entropy, and heat capacity. If the phonon DOS is adapted to the temperature and pressure of interest, there is often the expectation that harmonic phonons can be used to explain these thermodynamic quantities. Harmonic phonons are noninteracting eigenstates of atom displacements in a periodic crystal. Without phonon-phonon interactions, individual phonons are persistent. This remains true in "quasiharmonic theory," where harmonic phonon frequencies shift with changes in volume. Phonon-phonon interactions originate with "pure anharmonicity," caused by terms in the crystal Hamiltonian with cubic and quartic dependences on atom displacements. These interactions cause phonons to have finite lifetimes and hence a broadening of their energy spectra. Phonon-phonon interactions are essential to phonon transport properties, which are of technological importance as thermal conductivity.

The thermal conductivity of silicon has been studied extensively, motivated in part by the wide range of silicon-based

\footnotetext{
*dennis.s.kim@icloud.com

†btf@caltech.edu
}

technologies, including thermoelectrics, semiconductors, electronics, nanomechanics, and photovoltaics [1-10]. Historically, the measured thermal conductivity of silicon was used as a standard owing to the high purity and availability of silicon crystals. The thermal conductivity of silicon was measured directly with a radial heat flow apparatus [11,12]. More modern experimental techniques include the time and frequency-domain transient thermoreflectance methods and mean free path spectroscopy for both bulk and thin-film samples $[13,14]$.

The theory of thermal conductivity based on phonon anharmonicity traces back to the microscopic model developed by Peierls in 1929 [15], in which phonon-phonon interactions shorten the phonon mean free path for thermal transport. With advances in computational methods, detailed calculations of thermal conductivity have become practical in recent years. The lattice thermal conductivity is calculated by solving iteratively the Boltzmann transport equation or using the Green-Kubo method with either classical potentials or potentials derived from $a b$ initio methods [16-21]. From prior work on silicon, both classical forces and ab initio potentials with many-body perturbation theory have predicted the correct temperature dependence of the thermal conductivity $[17,19,22,23]$.

Thermal conductivity is a quantity averaged over numerous (of the order of Avogadro's number) individual phonon-phonon interactions, and the averaging masks many 
details. Experimental measurements on individual phonons offer more physical insight. For example, previous studies on silicon show that thermal shifts of phonon frequencies are not accurately described by the quasiharmonic model, even though it provides conventional explanations of thermal expansion [24,25]. To assess thermal conductivity, the thermal phonon lifetimes are essential. These connect to theory through the anharmonic corrections to the phonon self-energy, $\Sigma=\Delta+i \Gamma$, which give the thermal shifts as $\Delta$ and lifetimes of the phonons through $1 / 2 \Gamma$.

The inelastic scattering of thermal neutrons has been the workhorse method for measuring phonon dispersion curves in crystals. Historically, it has been a challenge to measure more than a few phonon spectral line shapes with triple-axis neutron spectrometers at reactor sources. The basic problem is low neutron flux combined with the need to make individual energy scans at single points in $\vec{Q}$ space. Other difficulties arise from instrument resolution and, sometimes, the accessibility of some combinations of $\vec{Q}$ and $E$. Nevertheless, triple-axis methods are valuable, and challenging measurements of multiple phonons have been performed [26-32].

Here we report work on phonon line shapes of silicon that benefited from the high neutron flux from a pulsed spallation neutron source and a technique using a position-sensitive detector array that covered approximately $\pi$ steradians around the sample. After correcting the measured data at temperature for the multiphonon background in the different Brillouin zones and "folding" the data from higher zones into the first, we obtained accurate spectral intensities of phonon dispersions at elevated temperatures. Fitting these dispersions can give both phonon frequencies and linewidths. A previous study reported how the average frequencies changed with temperature and assessed the physics of thermal expansion [24]. The present study is a report on the temperature dependence of phonon linewidths, which are used to interpret the thermal conductivity of silicon. Although some challenges with instrument resolution still remain, the thermal trends of phonon linewidths were obtained successfully. These thermal trends compared well with ab initio calculations of phonon spectra on supercells of silicon. These supercell methods gave parameters that were used with the Boltzmann transport equation to calculate the thermal conductivity of silicon at elevated temperature.

\section{METHODS}

\section{A. Neutron scattering}

Inelastic neutron scattering spectra on a single crystal of silicon were measured with the ARCS time-of-flight spectrometer at Oak Ridge National Lab as described previously [24]. The time-of-flight neutron data included multiple data sets from 200 rotations in increments of $0.5^{\circ}$ about the vertical [110] axis, reduced to create the four-dimensional $S(\vec{Q}, \varepsilon)$ using modern software tools $[33,35]$. Background corrections included subtraction of the multiphonon scattering, calculated in the incoherent approximation for the appropriate $\vec{Q}$ and $T$. A secondary data reduction process consisted of "folding" the entire $S(\vec{Q}, \varepsilon)$ data set into an irreducible wedge in the first Brillouin zone. Phonon intensities were then fitted as
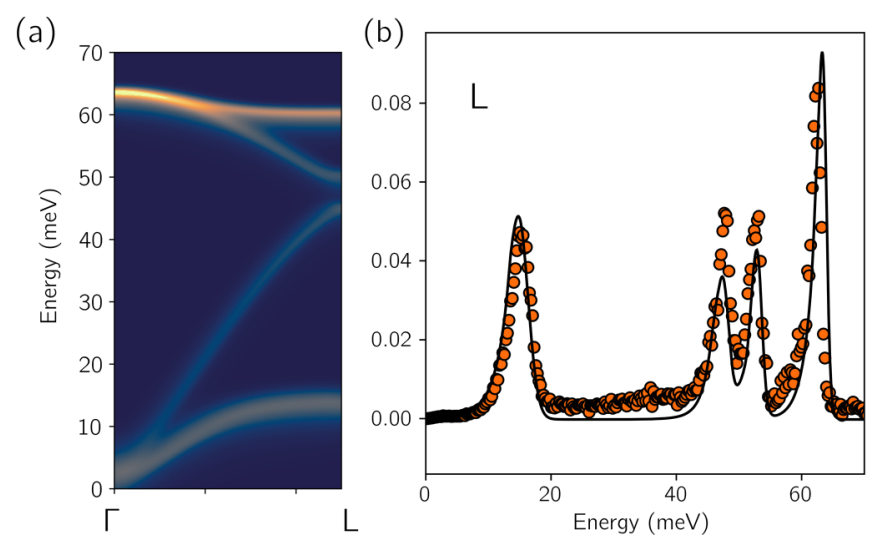

FIG. 1. Phonon intensities simulated for the operating conditions of the ARCS spectrometer. (a) $S(Q, \varepsilon)$ along the $\Gamma-L$ direction. (b) Phonon spectrum at the $L$ point from a MCVINE simulation (black line) and experiment (orange circles).

described previously, and changes in phonon linewidths at temperatures above $100 \mathrm{~K}$ were determined by tracking the difference in FWHM of the phonon peak measured at $100 \mathrm{~K}$ with the same $\vec{q}$ and branch.

\section{B. Monte Carlo simulations of experimental spectra}

To calculate the resolution of the ARCS instrument in $\vec{Q}$ and $\varepsilon$, we performed Monte Carlo Virtual Neutron Experiment (MCVINE) simulations [36] with the parameters of our experiment. This Monte Carlo neutron ray-tracing software incorporates the times when neutrons of different energies and angles are emitted from the decoupled ambient-temperature water moderator and accounts for the effects of the guide and choppers on the incident and scattered neutron profile in angle, position, and time. A set of sharp phonon dispersions of silicon gave the probabilities to deflect the incident neutrons based on temperature and momentum transfer. Figure 1(a) shows an example of a simulated slice of $S(\vec{Q}, \varepsilon)$. Figure 1(b) shows the peak asymmetry caused by the neutron moderator. While this spectrum is similar to experimental spectra shown later, the peaks are narrower, and the deconvolution of the calculated instrument resolution proved impractical. We therefore report changes in linewidth with temperature. With better computer algorithms, the direct measure of phonon lifetimes may be possible in the future.

\section{Computation}

$A b$ initio calculations were performed with VASP [37-40]. The projector augmented-wave formalism with the exchangecorrelation energy calculated with the Armiento and Mattson functional published in 2005 was used [41-43]. All calculations used $5 \times 5 \times 5$ supercells with a kinetic-energy cutoff of $500 \mathrm{eV}$ and a $3 \times 3 \times 3 \vec{Q}$-point grid. All calculations were converged to within $1 \mathrm{meV} /$ atom.

Phonon dispersions for anharmonic phonons at finite temperatures were calculated with the stochastically initialized temperature-dependent effective potential method (s-TDEP) described previously $[44,45]$. Finite-temperature phonon dispersions of silicon were calculated by fitting first-principles 
forces on atoms in the supercell to a model Hamiltonian,

$$
\begin{aligned}
H= & U_{0}+\sum_{i} \frac{\mathbf{p}_{i}^{2}}{2 m}+\frac{1}{2} \sum_{i j \alpha \beta} \Phi_{i j}^{\alpha \beta} u_{i}^{\alpha} u_{j}^{\beta} \\
& +\frac{1}{3 !} \sum_{i j k \alpha \beta \gamma} \Phi_{i j k}^{\alpha \beta \gamma} u_{i}^{\alpha} u_{j}^{\beta} u_{k}^{\gamma} .
\end{aligned}
$$

The forces on atoms were generated using density functional theory with various configurations of displaced atoms by a stochastic sampling of a canonical ensemble, with Cartesian displacements $u_{i}^{\alpha}$ normally distributed around the mean thermal displacement as

$$
u_{i}^{\alpha}=\sum_{\lambda} \frac{\epsilon_{\lambda}^{i \alpha} c_{\lambda}}{\sqrt{m_{i}}} \sqrt{-2 \ln \xi_{1}} \sin \left(2 \pi \xi_{2}\right) .
$$

The factor $c_{\lambda}$ is based on thermal amplitudes of the normal mode $\lambda$, with eigenvector $\epsilon_{\lambda}$ and frequency $\omega_{\lambda}$ [46-48],

$$
c_{\lambda}=\sqrt{\frac{\hbar\left(2 n_{\lambda}+1\right)}{2 \omega_{\lambda}}},
$$

and $\xi_{1}$ and $\xi_{2}$ are random numbers between 0 and 1 . The phonon occupancy was set by the Planck distribution, $n_{\lambda}=$ $\left(e^{\beta \hbar \omega_{\lambda}}-1\right)^{-1}$. In addition, the nuclear quantum effect (zero point amplitude) can be turned off by removing the 1 in Eq. (3).

Using thermal displacements from Eqs. (2) and (3), the temperature-dependent effective potential method (TDEP) $[44,45]$ was used to fit the parameters of model Hamiltonian, Eq. (1), in what we call the stochastically initialized temperature-dependent effective potential method. This method requires fewer computational resources than ab initio molecular-dynamics (AIMD), replacing AIMD with a Monte Carlo sampling of atomic positions and momentum near equilibrium positions $[44,46]$.

For all calculations, temperature-dependent equilibrium constants were used at each finite temperature and obtained through the minimization of the free energy,

$$
\begin{aligned}
F(T, V)= & E(T, V)+\sum_{\mathbf{q}, k}\left(\frac{\hbar \omega_{k}(\mathbf{q}, V, T)}{2}\right. \\
& \left.+k_{\mathrm{B}} T \ln \left(1-e^{-\hbar \omega_{k}(\mathbf{q}, T, V) / k_{\mathrm{B}} T}\right)\right) .
\end{aligned}
$$

In quasiharmonic theory, the temperature effects are approximated by the volume expansion. In contrast, the s-TDEP calculations minimize the free energy for temperature and volume simultaneously, therefore including pure anharmonicities and quasiharmonic effects [24]. Independently, calculations with a quasiharmonic model were performed as described previously [25]. Phonon dispersions from the quasiharmonic model were also used to provide the atom displacements of Eqs. (2) and (3) in the first iteration of s-TDEP.

\section{Phonon self-energies}

The phonon self-energy correction from many-body interactions $\Sigma_{\lambda}$ has real and imaginary parts,

$$
\Sigma_{\lambda}=\Delta_{\lambda}+i \Gamma_{\lambda}
$$

The phonon lifetimes $\left\{\tau_{\lambda}\right\}$ are related to the imaginary part of the self-energy as $\Gamma_{\lambda}=\left(2 \tau_{\lambda}\right)^{-1}$. The imaginary part of the self-energy is [49]

$$
\begin{aligned}
\Gamma_{\lambda}(\Omega)= & \frac{\hbar \pi}{16} \sum_{\lambda^{\prime} \lambda^{\prime \prime}}\left|\Phi_{\lambda \lambda^{\prime} \lambda^{\prime \prime}}\right|^{2} \\
& \times\left\{\left(n_{\lambda^{\prime}}+n_{\lambda^{\prime \prime}}+1\right) \delta\left(\Omega-\omega_{\lambda^{\prime}}-\omega_{\lambda^{\prime \prime}}\right)+\left(n_{\lambda^{\prime}}-n_{\lambda^{\prime \prime}}\right)\right. \\
& \left.\times\left[\delta\left(\Omega-\omega_{\lambda^{\prime}}+\omega_{\lambda^{\prime \prime}}\right)-\delta\left(\Omega+\omega_{\lambda^{\prime}}-\omega_{\lambda^{\prime \prime}}\right)\right]\right\} . \quad(6)
\end{aligned}
$$

$\Omega$ is the probing energy ( $\hbar \Omega=E$ ), and the $\delta$ functions conserve energy and momentum. The sum is over all possible three-phonon interactions between phonon modes. $\Phi_{\lambda \lambda^{\prime} \lambda^{\prime \prime}}$ is a three-phonon matrix element and is the Fourier transform of the third-order component of the interatomic potential,

$$
\begin{aligned}
\Phi_{\lambda \lambda^{\prime} \lambda^{\prime \prime}}= & \sum_{i j k} \sum_{\alpha \beta \gamma} \frac{\epsilon_{\lambda}^{i \alpha} \epsilon_{\lambda^{\prime}}^{j \beta} \epsilon_{\lambda^{\prime \prime}}^{k \gamma}}{\sqrt{m_{i} m_{j} m_{k}} \sqrt{\omega_{\lambda} \omega_{\lambda^{\prime}} \omega_{\lambda^{\prime \prime}}}} \\
& \times \Phi_{i j k}^{\alpha \beta \gamma} e^{i \vec{q} \cdot \vec{r}_{i}+i \vec{q}^{\prime} \cdot \vec{r}_{j}+i \vec{q}^{\prime \prime} \cdot \vec{r}_{k}},
\end{aligned}
$$

where $\vec{r}_{i}$ is the lattice vector of atom $i$. The real part of the selfenergy is calculated with a Kramers-Kronig transformation of the imaginary part,

$$
\Delta(\Omega)=\frac{1}{\pi} \int d \omega \frac{\Gamma(\omega)}{\omega-\Omega} .
$$

$A b$ initio calculations of phonon-self energies from sTDEP provided second- and third-order force constants, but effects of fourth-order perturbations were accounted for with the temperature dependences of the second-order constants and $U_{0}$.

\section{E. Thermal conductivity}

The Boltzmann transport equation for nonequilibrium phonon occupancies $\tilde{n}_{\lambda}$,

$$
\frac{\partial \tilde{n}_{\lambda}}{\partial T} \vec{v} \cdot \overrightarrow{\nabla T}=\left.\frac{\partial \tilde{n}_{\lambda}}{\partial t}\right|_{c}
$$

has a collision term on the right that can be obtained from the probabilities for down scattering and up scattering of a phonon $\lambda$,

$$
\begin{aligned}
P_{\lambda \lambda^{\prime} \lambda^{\prime \prime}}^{\downarrow}= & \frac{\hbar^{2} \pi}{16 N} n_{\lambda}\left(n_{\lambda^{\prime}}+1\right)\left(n_{\lambda^{\prime \prime}}+1\right)\left|\Phi_{\lambda \lambda^{\prime} \lambda^{\prime \prime}}\right|^{2} \\
& \times \delta\left(\omega_{\lambda}-\omega_{\lambda^{\prime}}-\omega_{\lambda^{\prime \prime}}\right), \\
P_{\lambda \lambda^{\prime} \lambda^{\prime \prime}}^{\uparrow} & =\frac{\hbar^{2} \pi}{16 N} n_{\lambda} n_{\lambda^{\prime}}\left(n_{\lambda^{\prime \prime}}+1\right)\left|\Phi_{\lambda \lambda^{\prime} \lambda^{\prime \prime}}\right|^{2} \\
& \times \delta\left(\omega_{\lambda}+\omega_{\lambda^{\prime}}-\omega_{\lambda^{\prime \prime}}\right) .
\end{aligned}
$$

The difference in thermal factors causes $P_{\lambda \lambda^{\prime} \lambda^{\prime \prime}}^{\downarrow}$ to dominate at low temperatures.

Force constants from both experimental data and $a b$ initio calculations were used obtain $\Phi_{\lambda \lambda^{\prime} \lambda^{\prime \prime}}$ in Eqs. (6), (10), and (11) and were used as parameters for iterative solutions of the full Boltzmann transport equation [19]. The only inputs were the second- and third-order force constants and $T$. Momentum conservation is exactly fulfilled, and energy conservation was employed with the tetrahedron method [50]. A $90 \times 90 \times 90 \vec{q}$-point grid was used, and thermal conductivity was converged with respect to $q$-point grid density to 
within $0.01 \%$. Pure anharmonicity from phonon-phonon interactions and scattering from the natural isotope distribution were included in calculating the thermal conductivity [51]. To identify the contributions of the individual phonon frequencies to the thermal conductivity, we calculated the spectral thermal conductance tensor $\kappa_{\lambda, \alpha \beta}(\omega)$ for each phonon branch $\lambda$, relating a $\beta$-direction applied temperature gradient to the thermal current in the $\alpha$ direction [52,53].

\section{RESULTS}

Four-dimensional scattering functions $S(\vec{Q}, \varepsilon)$ were obtained by reducing inelastic neutron scattering data at different temperatures. The methods included corrections for multiphonon background and "folding" into the first Brillouin zone. The reduced phonon spectra of Fig. 2(a) show bright intensity at the energies and wave vectors of phonon excitations. Following traditional presentations of phonon dispersion curves, we show intensities only along crystallographic directions of high symmetry. With temperature, phonon centroid energies soften (decrease in energy), as shown in Fig. 3(a) and presented previously in [24]. On average, the softening is proportional to the phonon energy, so the phonon DOS is selfsimilar with increasing temperature, as noted earlier [25]. The observed thermal trends are in good agreement with previous results where available $[12,54,55]$. Examples of fitted phonon peaks are shown in Figs. 2(b)-2(f). The longitudinal acoustic (LA) mode at $\vec{Q}=(0.75,0.25,0.25)$ is shown in Fig. 2(b), and the fit to an individual peak over the width of the box in Fig. 2(b) is shown in Fig. 2(c). Typical phonons and fits are shown in Figs. 2(e) and 2(f) for several temperatures for $\vec{Q}$ points at $(0.925,0.225,0.125),(0.725,0.325,0.225)$, and $(0.475,0.475,0.275)$. The peak broadenings are caused by both instrument resolution (as shown in Fig. 1) and the reduction of phonon lifetimes from pure anharmonicity. Changes with temperature of linewidths of typical phonons are shown in Fig. 3(b). The changes in linewidth are the difference between the phonon FWHM at $T$ and at $100 \mathrm{~K}$ (i.e., $2 \Gamma_{\mathrm{T}}-2 \Gamma_{100 \mathrm{~K}}$ ). Changes in linewidths at 300, 900, 1200, and $1500 \mathrm{~K}$ are thermal broadening of phonons since the instrument resolution is unchanged with temperature.

We fit over 500 phonon modes throughout the Brillouin zone. The s-TDEP method, even in its first iteration that used quasiharmonic phonon dispersions, showed good agreement with measured changes in phonon linewidths throughout the Brillouin zone. For the longitudinal acoustic modes and optical phonons, the calculations slightly underestimated the experimental thermal broadenings.

Figure 4 shows phonon linewidth broadenings throughout the Brillouin zone for four temperatures. Experimental linewidth broadenings are shown in Fig. 4(a), and s-TDEP broadenings are shown in Fig. 4(b). The differences in broadenings between experiments and s-TDEP calculations increase with temperature, becoming $15 \%$ at $1500 \mathrm{~K}$. Averaging all phonon energy shifts and broadenings over the Brillouin zone gives the results of Fig. 5 .

Using both the second- and third-order interatomic force constants, the Boltzmann transport equation was solved for different temperatures as described in Sec. II. We calculated total and mode-dependent spectral thermal conductivity, as shown in Figs. 6 and 7. We see that most of the heat is carried by low-energy phonons. Optical modes carry $5 \%$ of heat at $300 \mathrm{~K}$ and $8 \%$ at $1500 \mathrm{~K}$ (Fig. 6). The total thermal conductivity and its trend with temperature are in good agreement with experimental values (Fig. 7).

\section{DISCUSSION}

Thermophysical properties such as free energy, thermal expansion, and thermal conductivity are cumulative effects from numerous processes of phonon occupancy and phononphonon interactions. Thermophysical calculations that start with averaged quantities as inputs can give successful results, but some of their physical interpretations may be risky. Replacing a distribution of individual processes with its average loses the rich variation of phonon-phonon interactions. Individual processes need not follow the average behavior with temperature or volume. For example, it is possible to calculate the thermal expansion of silicon with quasiharmonic or anharmonic theory, and both give adequate results for this macroscopic quantity [24]. The two approaches differ significantly in their predictions of the thermal shifts of the centroids of phonon modes and usually disagree on the sign of the thermal shift.

In the 60 years since Brockhouse developed the tripleaxis spectrometer [56] inelastic neutron scattering has been used to measure phonon dispersions in single crystals. These measurements give simple data structures showing neutron intensity at individual energy transfers $\hbar \omega$ at a single momentum transfer $\hbar \vec{Q}$. Today, there is rapid development of single-crystal techniques with direct-geometry chopper spectrometers at spallation neutron sources. These measurements give arrival times of neutrons in detector pixels and can be reduced to measurements at many simultaneous $\vec{Q}$ and $\omega$. Improvements in geometrical efficiency may be of the order of $10^{4}$, and the high neutron flux allows for much more complete data sets in shorter times. This is arguably less advantageous for the average energies of phonons because lattice dynamics models, such as the Born-von Kármán model, can be used to assess the energies of phonons that are not measured. The linewidths of phonons cannot be modeled simply, however, so linewidth measurements for all phonons have a higher priority for understanding thermal transport. The line shapes of individual phonons are obtained with high precision, as shown in some detail in Fig. 2.

Monte Carlo simulations of instrument resolution [Fig. 1(b)] were not sufficiently accurate to warrant deconvoluting the measured phonon peaks. Nevertheless, the trends of the experimental linewidths with temperature (and phonon scattering lifetimes) are in good agreement with thermal trends from the $a b$ initio calculations. For phonon peaks on sloping dispersion curves, there are risks of broadening if the energy spectra are obtained from large volumes in reciprocal space, but experimental integrations were narrow [Fig. 2(a)], and phonons from sloping dispersions showed no systematic problems. Each integration was taken over a $0.0025 \AA^{-3}$ volume in $\vec{Q}$ space, and we find the phonon thermal broadenings do not change significantly even for phonon modes with large group velocities (see the Supplemental Material [34]). For 
(a)

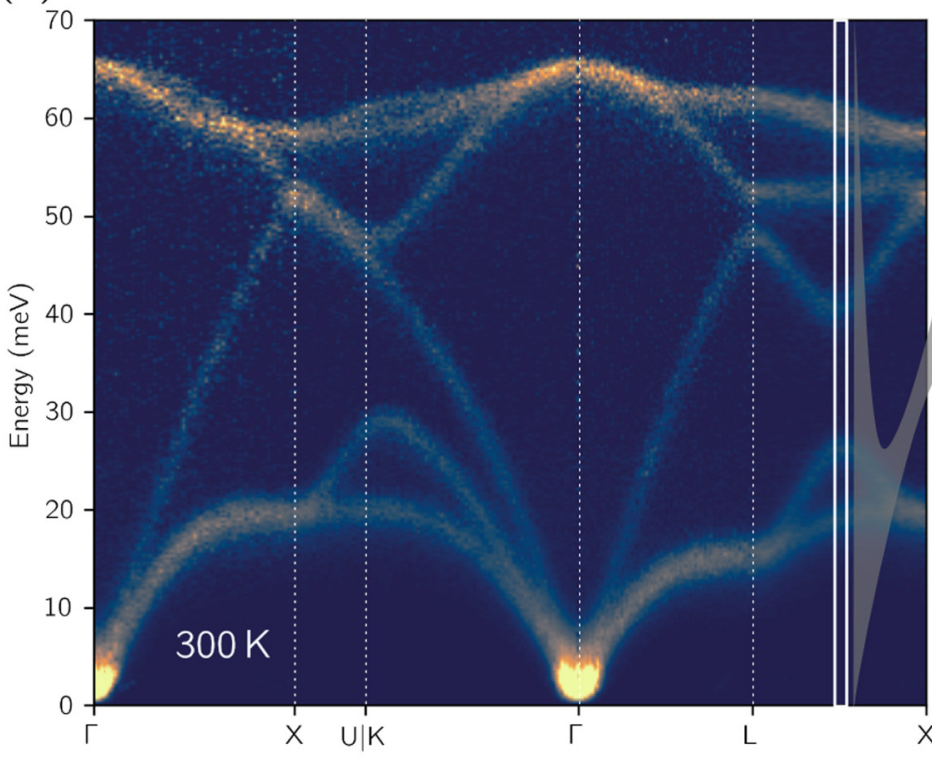

(d)
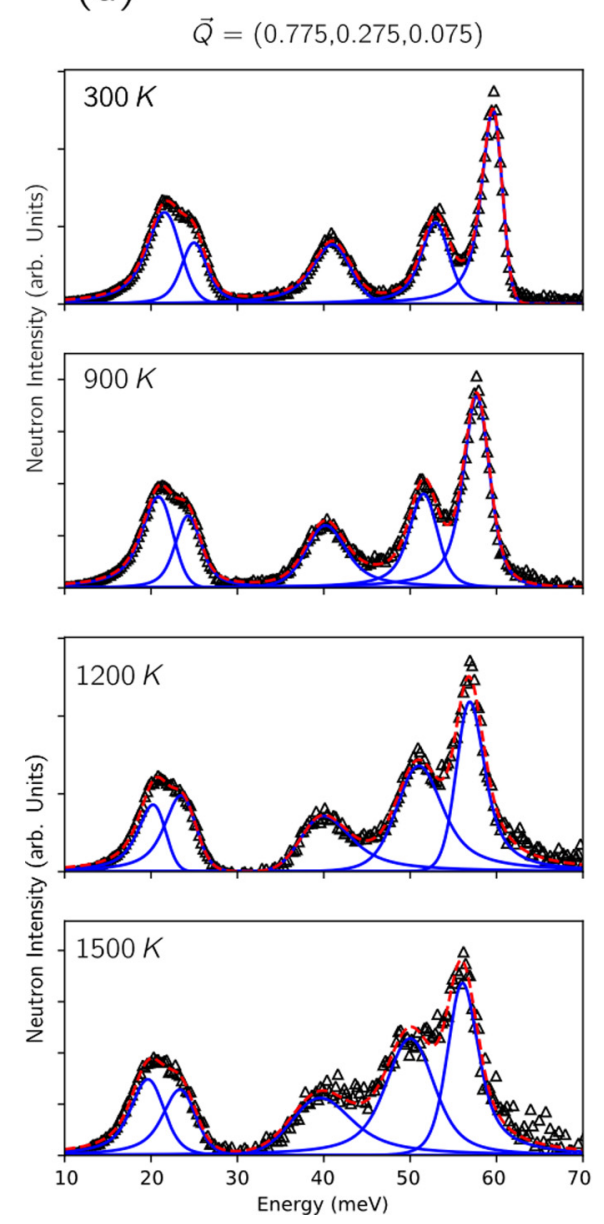

(e)
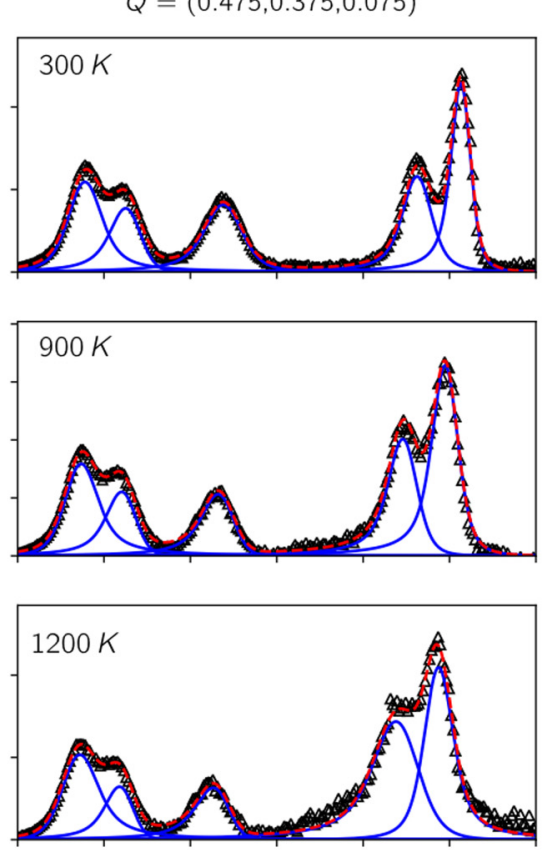

(b)

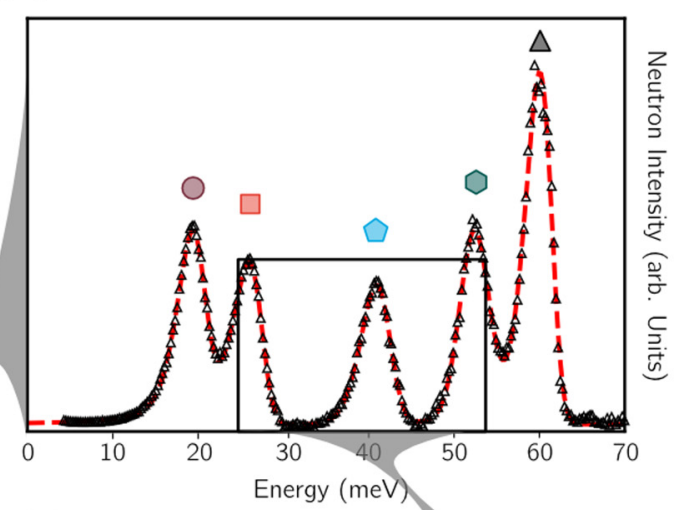

(c)

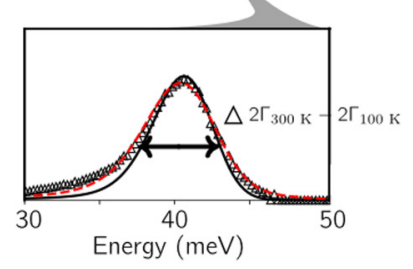

(f)
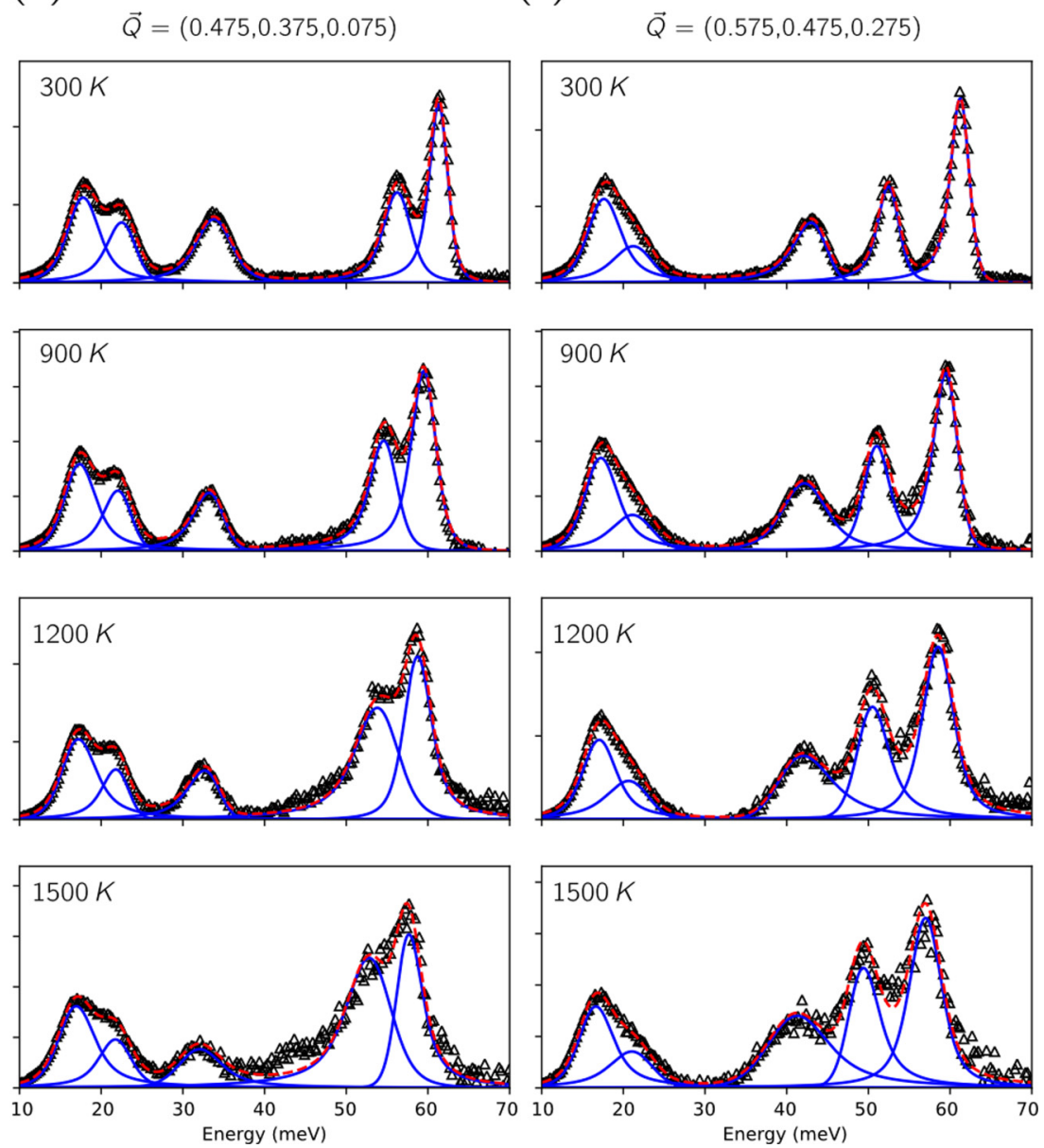

FIG. 2. Phonon dispersions and individual peaks from INS data from silicon at $300 \mathrm{~K}$. (a) Example of experimental phonon dispersions along directions of high symmetry. (b) Phonon scattering spectra at $\vec{Q}=(0.75,0.25,0.25)$ r.l.u., which is along the vertical cut shown in panel a. (c) Line shape of longitudinal acoustic phonon peak at $\vec{Q}=(0.75,0.25,0.25)$ r.l.u. Experimental data are triangle markers, and dashed red lines are best fits in both (b) and (c) for $300 \mathrm{~K}$. The black curve in (c) is the best fit for the longitudinal acoustic phonon peak at $\vec{Q}=(0.75,0.25,0.25)$ r.l.u. at $100 \mathrm{~K}$. Phonon peaks at $300,900,1200$, and $1500 \mathrm{~K}$ at $\vec{Q}$ points (d) $(0.775,0.275,0.075),(\mathrm{e})(0.475,0.375,0.075)$, and (f) $(0.575,0.475,0.275)$. Experimental data are triangle markers, and dashed red lines are the best fit, with individual peak fits shown as blue lines. 

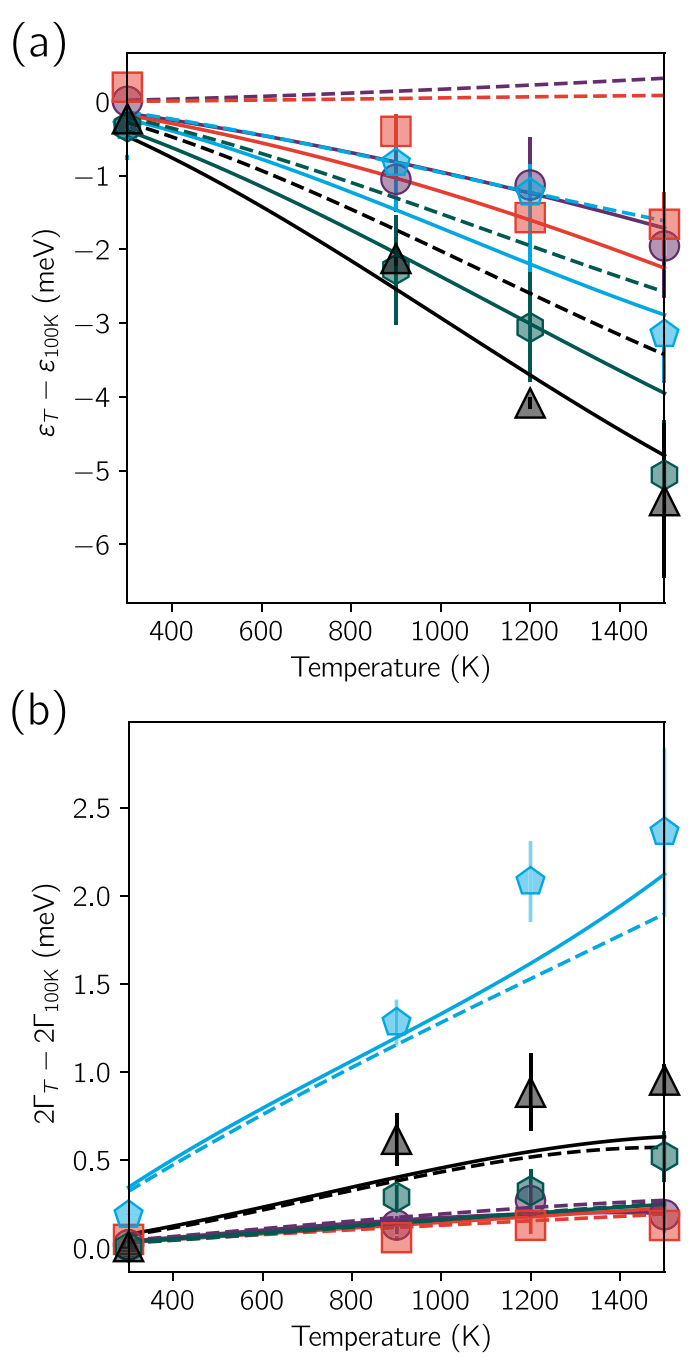

FIG. 3. (a) Phonon energy shifts and (b) energy broadenings at $\vec{Q}=(0.75,0.25,0.25)$ versus temperature. Experimental results are markers that correspond to the peaks shown in Fig. 2(b). Solid lines are from $a b$ initio calculations with anharmonicity. Dashed lines are from the quasiharmonic approximation used in the first iteration of s-TDEP.

hundreds of phonon modes throughout the Brillouin zone, the largest discrepancies were found around $47 \mathrm{meV}$. These discrepancies could be due to the intricate set of dispersion curves near high-symmetry points. Previous Raman measurements of thermal trends for the optical mode at the $\Gamma$ point agree well with our results and show thermal rates of change that differ by $8 \%[27,57,58]$

Both the experimental and computational work showed large thermal broadening of the LA modes (Fig. 4), indicating short phonon lifetimes. This helps explain why the transverse acoustic modes are more important for thermal conductivity than the LA, in spite of the higher group velocity for LA modes (Fig. 6). Only a little heat is carried by optical phonons because their group velocities are low, and their lifetimes are relatively short.

The cubic anharmonicity is the first term in the Hamiltonian with an imaginary component that gives phonon lifetime broadening, but it may not suffice at high temperatures. We (a)

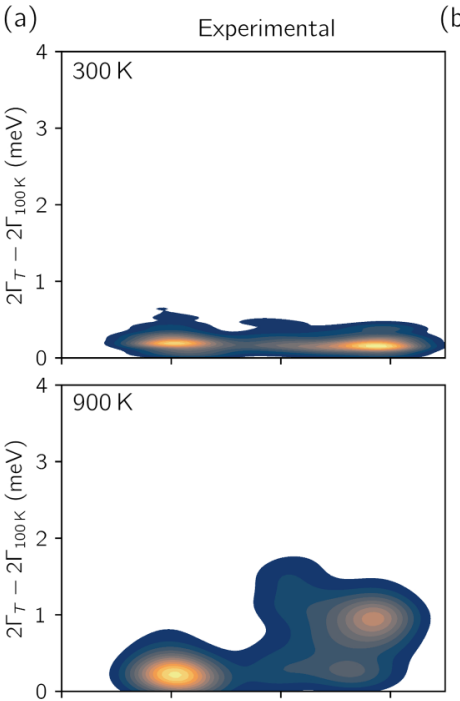

(b)
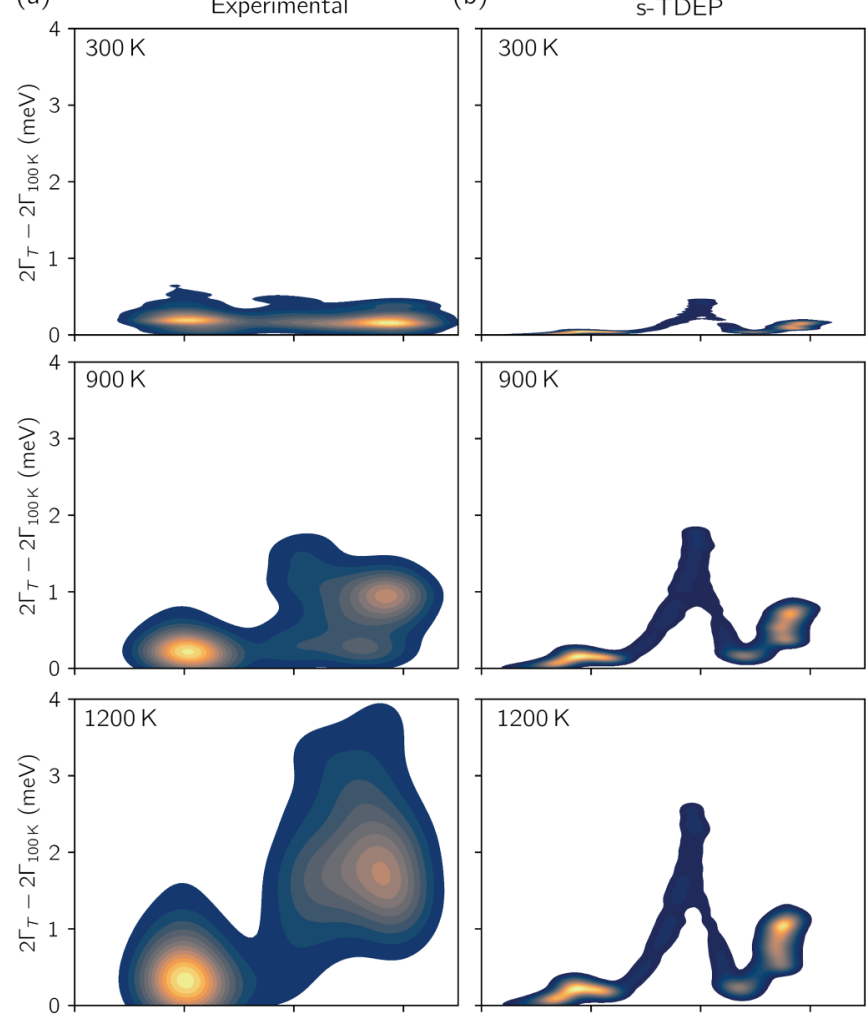

$1200 \mathrm{~K}$
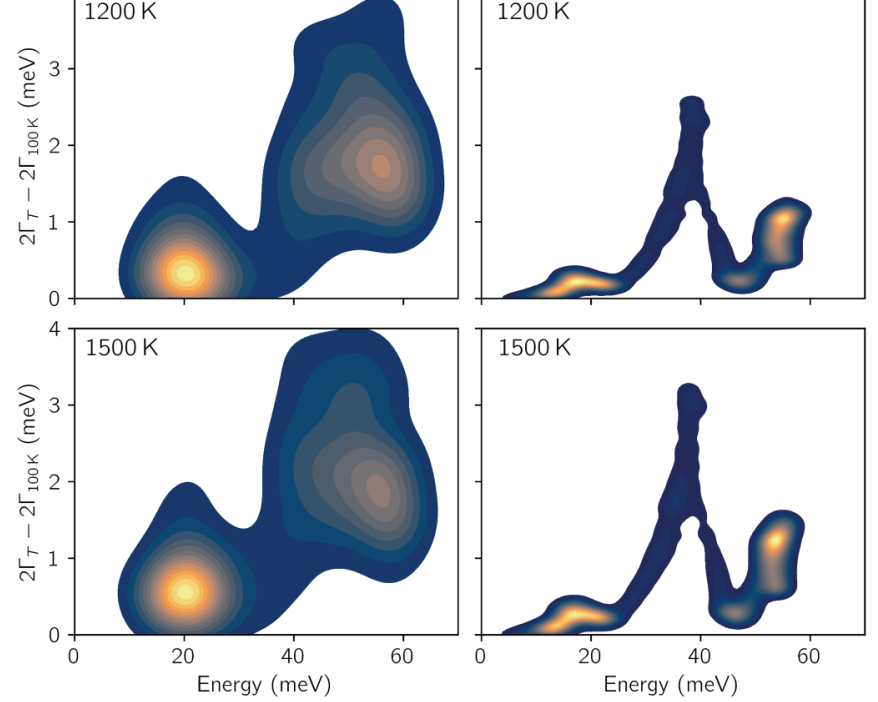

FIG. 4. Increase in phonon linewidth from linewidth at $100 \mathrm{~K}$ versus phonon energy at $300,900,1200$, and $1500 \mathrm{~K}$ as labeled. (a) Experimental linewidths and (b) s-TDEP calculated linewidths. Data are from phonons throughout the Brillouin zone.

found some differences between experimental and calculated linewidths of individual phonons at $1500 \mathrm{~K}$ [Fig. 3(b)] but less discrepancy in the shift and broadening averaged over the Brillouin zone (Fig. 5). There are perhaps similar deviations in the calculated thermal conductivity (Fig. 7). It appears that the effects of anharmonicity were slightly underestimated by s-TDEP. Perhaps more surprising, though, is the overall success of this computational scheme over such a wide range of temperatures. Even the first iteration of s-TDEP, which uses quasiharmonic phonons for the displacements $\left\{u_{i}^{\alpha}\right\}$ of Eq. (1), gives phonon shifts and broadenings that are in reasonable agreement with the converged s-TDEP calculations, except at the highest temperatures.

Some of this success of s-TDEP may arise from a peculiarity of phonons in silicon. We reported earlier that the phonon DOS of silicon is nearly self-similar with increasing temperature, with the full spectrum contracting approximately uniformly with increasing temperature [25]. This average 

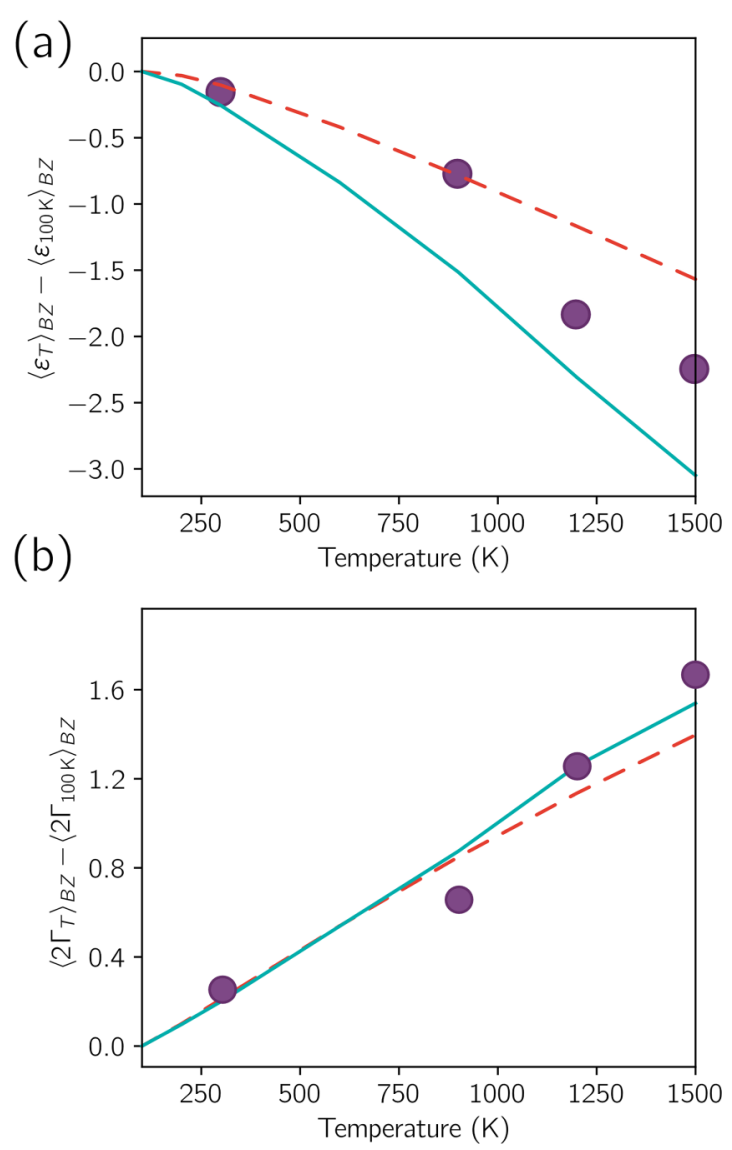

FIG. 5. Temperature-dependent averages over the Brillouin zone of phonon (a) energies and (b) linewidths. Purple circles are experimental data, the solid teal line is from ab initio calculations with s-TDEP, and the red dashed line is the first iteration of s-TDEP using quasiharmonic phonons.

self-similarity of the acoustic and optic modes allows the different three-phonon processes to maintain similar weights over a wide range of temperature. If the effect of temperature is primarily to rescale the energies of the phonon dispersions, the three-phonon processes allowed by the kinematics of energy and momentum conservation will be the same. At high temperatures where Eqs. (10) and (11) are comparable, we expect similar shapes for the phonon linewidth plots of Fig. 4, and this is approximately true at temperatures of $900 \mathrm{~K}$ and higher. This also explains why there are only small differences between the results from s-TDEP in its first iteration with quasiharmonic phonons and after iterating to obtain more accurate phonon energies and thermal broadening from anharmonicity. These two calculations also gave very similar group velocities and similar phonon lifetimes, with some discrepancies for higher-energy modes $(>30 \mathrm{meV})$. We found that thermal expansion does not significantly alter the results from constant-volume calculations. The contribution from the higher-energy modes is decreased as the overall softening heightens the low transverse mode contribution to overall thermal conductivity.

For silicon from 100 to $1500 \mathrm{~K}$ the energy broadenings of phonon peaks were generally smaller than for other materials at temperatures of equivalent fractions of their melting (a)

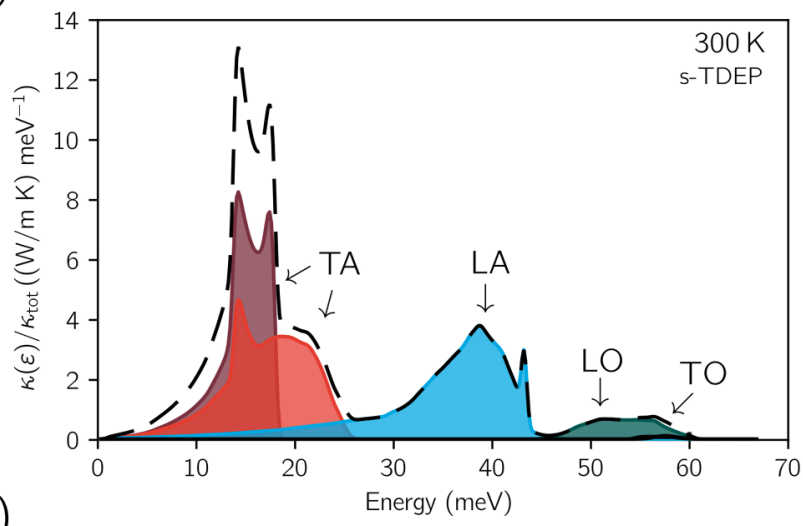

(b)

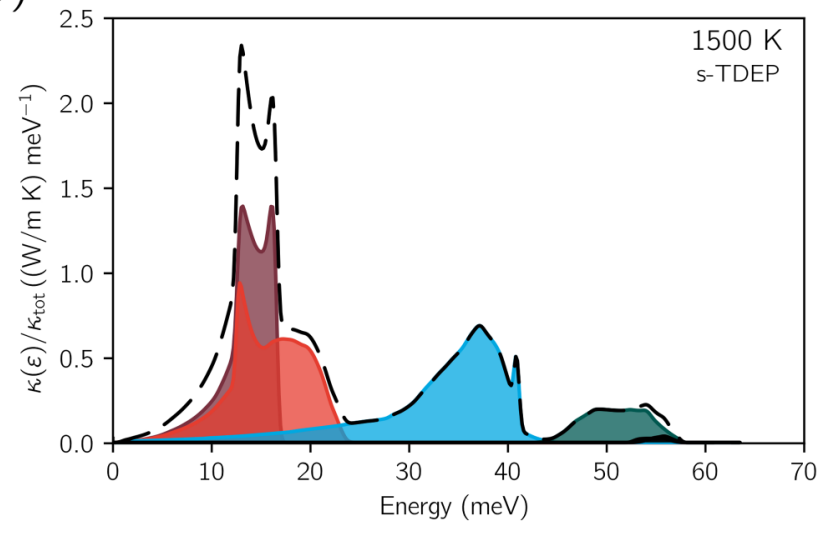

FIG. 6. Calculated spectral thermal conductivity $\kappa(\varepsilon) / \kappa_{\text {tot }}$ at (a) $300 \mathrm{~K}$ and (b) $1500 \mathrm{~K}$. Total spectral thermal conductivity is shown by the black dashed line. Contributions from TA, LA, LO, and TO modes are labeled in (a).

temperature [32,59-61]. It could be argued that silicon is "less anharmonic" [61]. Nevertheless, from the present study of phonon line shapes and our previous study of phonon energy shifts [24], for silicon the deviations from harmonic behavior, although not large, are dominated by anharmonicity, not quasiharmonicity.

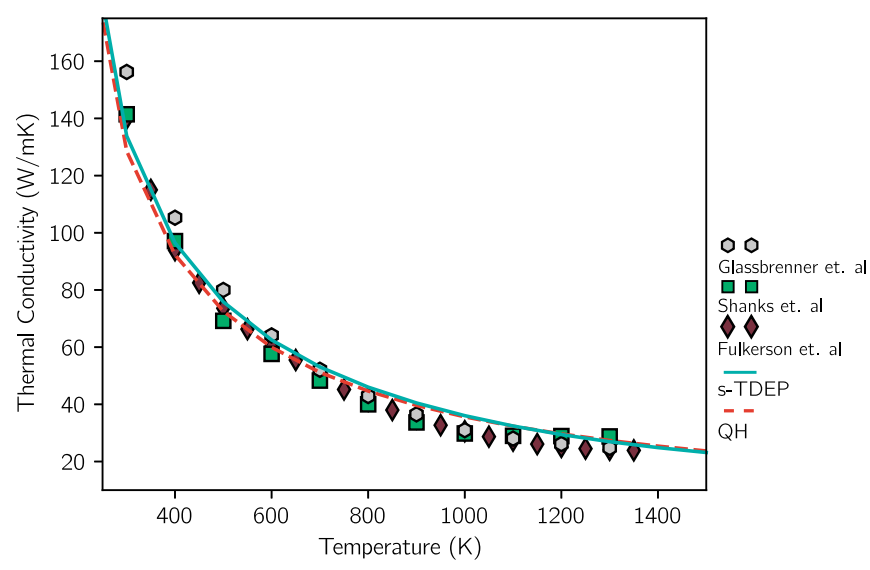

FIG. 7. Temperature-dependent thermal conductivity of silicon. Phonon anharmonic ab initio calculations (s-TDEP) are shown as a solid teal line, the s-TDEP in its first iteration with the quasiharmonic model is shown by the red dashed line, and experimental points are shown as markers [12,54,55]. 


\section{v. CONCLUSIONS}

Phonon spectral weight in the four-dimensional space of $\{\vec{Q}, \omega\}$ was measured and calculated for diamond cubic silicon at temperatures from 100 to $1500 \mathrm{~K}$. The energy linewidths of phonon peaks were obtained for hundreds of phonons in the first Brillouin zone. The changes in phonon broadening with temperature were in good agreement between computation and experiment, although the distributions of phonon linewidths in the experimental data were broader in experiment than in s-TDEP calculations. Discrepancies also appear at temperatures above $1000 \mathrm{~K}$. This suggests that the anharmonic effects are moderately larger than predicted with the present computations. Nevertheless, distributions of phonon widths versus phonon energy had similar shapes for computation and experiment. These distributions grew with temperature but maintained similar shapes, likely because of the uniform thermal softenings of the dispersion curves with temperature.

Calculations of thermal conductivity with the Boltzmann transport equation were in excellent agreement with experimental data. The spectral thermal conductivity showed that the transverse acoustic modes dominated the thermal con- ductivity. Contributions from other modes are non-negligible, and these contributions increase with temperature. The longitudinal acoustic modes had high group velocities but short lifetimes. The optical modes had low group velocities and short lifetimes. The spectral thermal conductivity changes moderately with temperature. The optical modes carry more heat at high temperatures, but no more than $10 \%$ of the total.

\section{ACKNOWLEDGMENTS}

Research at Oak Ridge National Laboratory's SNS was sponsored by the Scientific User Facilities Division, BES, DOE. This work used resources from NERSC, a DOE Office of Science User Facility supported by the Office of Science of the US Department of Energy under Contract No. DE-AC0205CH11231. Support from Swedish Research Council (VR) Program No. 637-2013-7296 is also gratefully acknowledged. Supercomputer resources were provided by the Swedish National Infrastructure for Computing (SNIC). This work was supported by the DOE Office of Science, BES, under Contract No. DE-FG02-03ER46055.
[1] L.-D. Zhao, S.-H. Lo, Y. Zhang, H. Sun, G. Tan, C. Uher, C. Wolverton, V. P. Dravid, and M. G. Kanatzidis, Ultralow thermal conductivity and high thermoelectric figure of merit in SnSe crystals, Nature (London) 508, 373 (2014).

[2] M. A. Green, J. Zhao, A. Wang, P. J. Reece, and M. Gal, Efficient silicon light-emitting diodes, Nature (London) 412, 805 (2001).

[3] A. I. Hochbaum, R. Chen, R. D. Delgado, W. Liang, E. C. Garnett, M. Najarian, A. Majumdar, and P. Yang, Enhanced thermoelectric performance of rough silicon nanowires, Nature 451, 163 (2008).

[4] J. Graetz, C. C. Ahn, R. Yazami, and B. Fultz, Highly reversible lithium storage in nanostructured silicon, Electrochem. SolidState Lett. 6, A194 (2003).

[5] Y. Cui, Z. Zhong, D. Wang, W. U. Wang, and C. M. Lieber, High performance silicon nanowire field effect transistors, Nano Lett. 3, 149 (2003).

[6] A. I. Boukai, Y. Bunimovich, J. Tahir-Kheli, J.-K. Yu, W. A. Goddard III, and J. R. Heath, Silicon nanowires as efficient thermoelectric materials, Nature (London) 451, 168 (2008).

[7] H. A. Atwater and A. Polman, Plasmonics for improved photovoltaic devices, Nat. Mater. 9, 205 (2010).

[8] B. Tian, X. Zheng, T. J. Kempa, Y. Fang, N. Yu, G. Yu, J. Huang, and C. M. Lieber, Coaxial silicon nanowires as solar cells and nanoelectronic power sources, Nature (London) 449, 885 (2007).

[9] L. Tsybeskov, S. P. Duttagupta, K. D. Hirschman, P. M. Fauchet, K. L. Moore, and D. G. Hall, Room-temperature photoluminescence and electroluminescence from Er-doped silicon-rich silicon oxide, Appl. Phys. Lett. 70, 1790 (1997).

[10] A. J. Minnich, M. S. Dresselhaus, Z. F. Ren, and G. Chen, Bulk nanostructured thermoelectric materials: Current research and future prospects, Energy Environ. Sci. 2, 466 (2009).
[11] P. D. Maycock, Thermal conductivity of silicon, germanium, III-V compounds and III-V alloys, Solid-State Electron. 10, 161 (1967).

[12] C. J. Glassbrenner and G. A. Slack, Thermal conductivity of silicon and germanium from $3 \mathrm{~K}$ to the melting point, Phys. Rev. 134, A1058 (1964).

[13] D. G. Cahill, P. V. Braun, G. Chen, D. R. Clarke, S. Fan, K. E. Goodson, P. Keblinski, W. P. King, G. D. Mahan, A. Majumdar, H. J. Maris, S. R. Phillpot, E. Pop, and L. Shi, Nanoscale thermal transport. II. 2003-2012, Appl. Phys. Rev. 1, 011305 (2014).

[14] A. J. Minnich, Determining Phonon Mean Free Paths from Observations of Quasiballistic Thermal Transport, Phys. Rev. Lett. 109, 205901 (2012).

[15] R. Peierls, Zur kinetischen Theorie der Wärmeleitung in Kristallen, Ann. Phys. (Berlin, Ger.) 395, 1055 (1929).

[16] G. Chen, Thermal conductivity and ballistic-phonon transport in the cross-plane direction of superlattices, Phys. Rev. B 57, 14958 (1998).

[17] D. A. Broido, A. Ward, and N. Mingo, Lattice thermal conductivity of silicon from empirical interatomic potentials, Phys. Rev. B 72, 014308 (2005).

[18] J. Garg, N. Bonini, B. Kozinsky, and N. Marzari, Role of Disorder and Anharmonicity in the Thermal Conductivity of Silicon-Germanium Alloys: A First-Principles Study, Phys. Rev. Lett. 106, 045901 (2011).

[19] D. A. Broido, M. Malorny, G. Birner, N. Mingo, and D. A. Stewart, Intrinsic lattice thermal conductivity of semiconductors from first principles, Appl. Phys. Lett. 91, 231922 (2007).

[20] N. Shulumba, O. Hellman, and A. J. Minnich, Intrinsic localized mode and low thermal conductivity of PbSe, Phys. Rev. B 95, 014302 (2017).

[21] A. Henry and G. Chen, High Thermal Conductivity of Single Polyethylene Chains Using Molecular Dynamics Simulations, Phys. Rev. Lett. 101, 235502 (2008). 
[22] T. Feng and X. Ruan, Quantum mechanical prediction of fourphonon scattering rates and reduced thermal conductivity of solids, Phys. Rev. B 93, 045202 (2016).

[23] K. Esfarjani, G. Chen, and H. T. Stokes, Heat transport in silicon from first-principles calculations, Phys. Rev. B 84, 085204 (2011).

[24] D. S. Kim, O. Hellman, J. Herriman, H. L. Smith, J. Y. Y. Lin, N. Shulumba, J. L. Niedziela, C. W. Li, D. L. Abernathy, and B. Fultz, Nuclear quantum effect with pure anharmonicity and the anomalous thermal expansion of silicon, Proc. Natl. Acad. Sci. U.S.A. 115, 1992 (2018).

[25] D. S. Kim, H. L. Smith, J. L. Niedziela, C. W. Li, D. L. Abernathy, and B. Fultz, Phonon anharmonicity in silicon from 100 to 1500 K, Phys. Rev. B 91, 014307 (2015).

[26] O. Delaire, J. Ma, K. Marty, A. F. May, M. A. McGuire, M.-H. Du, D. J. Singh, A. Podlesnyak, G. Ehlers, M. D. Lumsden, and B. C. Sales, Giant anharmonic phonon scattering in $\mathrm{PbTe}$, Nat. Mater. 10, 614 (2011).

[27] G. Lang, K. Karch, M. Schmitt, P. Pavone, A. P. Mayer, R. K. Wehner, and D. Strauch, Anharmonic line shift and linewidth of the Raman mode in covalent semiconductors, Phys. Rev. B 59, 6182 (1999).

[28] S. Narasimhan and D. Vanderbilt, Anharmonic self-energies of phonons in silicon, Phys. Rev. B 43, 4541 (1991).

[29] A. Debernardi, S. Baroni, and E. Molinari, Anharmonic Phonon Lifetimes in Semiconductors from Density-Functional Perturbation Theory, Phys. Rev. Lett. 75, 1819 (1995).

[30] J. H. Parker, D. W. Feldman, and M. Ashkin, Raman scattering by silicon and germanium, Phys. Rev. 155, 712 (1967).

[31] P. G. Klemens, Anharmonic decay of optical phonons, Phys. Rev. 148, 845 (1966).

[32] A. Glensk, B. Grabowski, T. Hickel, J. Neugebauer, J. Neuhaus, K. Hradil, W. Petry, and M. Leitner, Phonon Lifetimes Throughout the Brillouin Zone at Elevated Temperatures from Experiment and Ab Initio, Phys. Rev. Lett. 123, 235501 (2019).

[33] MANTID, http://dx.doi.org/10.5286/SOFTWARE/MANTID.

[34] See Supplemental Material at http://link.aps.org/supplemental/ 10.1103/PhysRevB.102.174311 for a figure that shows how the broadening in linewidth depends on the integration range in $Q$ space.

[35] O. Arnold, J. C. Bilheux, J. M. Borreguero, A. Buts, S. I. Campbell, L. Chapon, M. Doucet, N. Draper, R. Ferraz Leal, M. A. Gigg, V. E. Lynoh, A. Markvardsen, D. J. Mikkelson, R. L. Mikkelson, R. Miller, K. Palmer, P. Parker, G. Passons, I. G. Perring, P. F. Peterson, S. Ren, M. A. Reuter, A. T. Savici, J. W. Taylor, R. J. Taylor, R. Tolchenov, W. Zhen, and J. Zikovsky, Mantid-Data analysis and visualization package for neutron scattering and $\mu$ SR experiments, Nucl. Instrum. Methods Phys. Res., Sect. A 764, 156 (2014).

[36] J. Y. Y. Lin, H. L. Smith, G. E. Granroth, D. L. Abernathy, M. D. Lumsden, B. Winn, A. A. Aczel, M. Aivazis, and B. Fultz, MCViNE - An object oriented Monte Carlo neutron ray tracing simulation package, Nucl. Instrum. Methods Phys. Res., Sect. A 810, 86 (2016).

[37] G. Kresse and J. Furthmüller, Efficiency of ab-initio total energy calculations for metals and semiconductors using a plane-wave basis set, Comput. Mater. Sci. 6, 15 (1996).

[38] G. Kresse and J. Hafner, Ab initio molecular dynamics for liquid metals, Phys. Rev. B 47, 558 (1993).
[39] G. Kresse and J. Hafner, Ab initio molecular-dynamics simulation of the liquid-metal-amorphous-semiconductor transition in germanium, Phys. Rev. B 49, 14251 (1994).

[40] X. Gonze and C. Lee, Dynamical matrices, Born effective charges, dielectric permittivity tensors, and interatomic force constants from density-functional perturbation theory, Phys. Rev. B 55, 10355 (1997).

[41] R. Armiento and A. E. Mattsson, Functional designed to include surface effects in self-consistent density functional theory, Phys. Rev. B 72, 085108 (2005).

[42] A. E. Mattsson and R. Armiento, Implementing and testing the AM05 spin density functional, Phys. Rev. B 79, 155101 (2009).

[43] A. E. Mattsson, R. Armiento, J. Paier, G. Kresse, J. M. Wills, and T. R. Mattsson, The AM05 density functional applied to solids, J. Chem. Phys. 128, 084714 (2008).

[44] O. Hellman, P. Steneteg, I. A. Abrikosov, and S. I. Simak, Temperature dependent effective potential method for accurate free energy calculations of solids, Phys. Rev. B 87, 104111 (2013).

[45] O. Hellman and I. A. Abrikosov, Temperature dependent effective third order interatomic force constants from first principles, Phys. Rev. B 88, 144301 (2013).

[46] I. Errea, M. Calandra, and F. Mauri, Anharmonic free energies and phonon dispersions from the stochastic self-consistent harmonic approximation: Application to platinum and palladium hydrides, Phys. Rev. B 89, 064302 (2014).

[47] D. C. Wallace, Thermodynamics of Crystals (Dover, New York, 1998).

[48] M. T. Dove, Introduction to Lattice Dynamics (Cambridge University Press, Cambridge, 1993).

[49] A. A. Maradudin and A. E. Fein, Scattering of neutrons by an anharmonic crystal, Phys. Rev. 128, 2589 (1962).

[50] G. Lehmann and M. Taut, On the numerical calculation of the density of states and related properties, Physica Status Solidi B 54, 469 (1972).

[51] S.-i. Tamura, Isotope Scattering of Dispersive Phonons in Ge, Phys. Rev. B 27, 858 (1983).

[52] R. E. Peierls, Quantum Theory of Solids (Oxford University Press, Oxford, 2001).

[53] O. Hellman, Temperature dependent effective potential, https: //ollehellman.github.io.

[54] H. R. Shanks, P. D. Maycock, P. H. Sidles, and G. C. Danielson, Thermal conductivity of silicon from 300 to 1400 K, Phys. Rev. 130, 1743 (1963).

[55] W. Fulkerson, J. P. Moore, R. K. Williams, R. S. Graves, and D. L. McElroy, Thermal conductivity, electrical resistivity, and Seebeck coefficient of silicon from 100 to $1300^{\circ}$, Phys. Rev. 167, 765 (1968).

[56] B. N. Brockhouse and P. K. Iyengar, Normal Vibrations of Germanium by Neutron Spectrometry, Phys. Rev. 108, 894 (1957).

[57] J. Menéndez and M. Cardona, Temperature dependence of the first-order Raman scattering by phonons in $\mathrm{Si}, \mathrm{Ge}$, and $\alpha-\mathrm{Sn}$ : Anharmonic effects, Phys. Rev. B 29, 2051 (1984).

[58] R. Tsu and J. G. Hernandez, Temperature dependence of silicon Raman lines, Appl. Phys. Lett. 41, 1016 (1982).

[59] J. W. L. Pang, W. J. L. Buyers, A. Chernatynskiy, M. D. Lumsden, B. C. Larson, and S. R. Phillpot, Phonon Lifetime 
Investigation of Anharmonicity and Thermal Conductivity of $\mathrm{UO}_{2}$ by Neutron Scattering and Theory, Phys. Rev. Lett. 110, 157401 (2013).

[60] M. S. Bryan, J. W. L. Pang, B. C. Larson, A. Chernatynskiy,

D. L. Abernathy, K. Gofryk, and M. E. Manley, Impact of anharmonicity on the vibrational entropy and specific heat of $\mathrm{UO}_{2}$, Phys. Rev. Mater. 3, 065405 (2019).

[61] F. Knoop, T. A. R. Purcell, M. Scheffler, and C. Carbogno, Anharmonicity measure for materials, Phys. Rev. Mater. 4, 083809 (2020). 